\title{
ABNORMAL LYMPHATIC PHENOTYPE IN A CRISPR MOUSE MODEL OF THE HUMAN LYMPHEDEMA-CAUSING CONNEXIN47 R260C POINT MUTATION
}

\author{
D.J. Mustacich, R.I. Kylat, M.J. Bernas, R.J. Myles, J.A. Jones, J.D. Kanady, \\ A.M. Simon, T.G. Georgieva, M.H. Witte, R.P. Erickson, P.W. Pires
}

Department of Surgery (DJM,MJB,RJM,JAJ,MHW), Department of Pediatrics (RIK,RPE); Department of Physiology (JDK,AMS,PWP); GEMM Core (TGG), Bio5 Institute, University of Arizona, Tucson, AZ; TCU and UNTHSC School of Medicine (MJB), Fort Worth, TX, USA

\section{ABSTRACT}

Connexin proteins form gap junctions controlling exchange of ions and small molecules between cells and play an important role in movement of lymph within lymphatic vessels. Connexin47 (CX47) is highly expressed in lymphatic endothelial cells and CX47 missense mutations, i.e., R260C, cosegregate with primary lymphedema in humans. However, studies utilizing CX47 knockout mice have failed to demonstrate any lymphatic anomalies. To unravel the lymphatic consequences of expressing a mutant CX47 protein, we used CRISPR technology to create a mouse carrying a $C \times 47$ missense mutation (CX47R259C) equivalent to the human CX47R260C missense mutation associated with human primary lymphedema. Intradermal Evans Blue dye injection identified a 2-fold increase in regional lymph nodes in homozygous Cx47R259C mice compared to wildtype, particularly in the jugular region (4.8 \pm 0.4 and $2.0 \pm 0.0$, respectively, $p<0.01$ ). Associated lymphatic channels were increased in Cx47R259C mice and mesenteric lymph reflux occurred in homozygous Cx47R259C mice but not in wildtype. Contractility of superficial cervical lymphatics, assessed by pressure myography, was reduced in homozygous CX47R259C mice compared to wildtype.
In conclusion, our data are the first to demonstrate a role for the CX47 protein in lymphatic anatomy and function. This phenotype is similar to that found with other valve deficient mouse mutants, e.g., in Foxc2. Of significance, this study is the first to use CRISPR technology to develop a pre-clinical model of primary lymphedema and demonstrates the importance of distinguishing between lack of and presence of mutant protein when developing clinically relevant animal models for translation of pre-clinical findings.

Keywords: hereditary lymphedema, lymphatic contractility, gap junctions, connexins, CRISPR mouse, genotype-phenotype correlations.

Lymphedema, or the excessive accumulation of interstitial fluid (ISF) in tissues, is a painful condition that affects millions of people worldwide (1-3). Although lymphedema is a multifactorial disease and can be secondary to other disorders, a characteristic of the condition is impaired lymphatic drainage, which can be caused by a physical blockage or reduced pumping function of collecting lymphatic vessels (4). Efficient ISF drainage and lymph pumping, in turn, requires rhythmic and coordinated contraction cycles of 
lymphangions (inter-valve segments of lymphatic segments), in conjunction with properly functioning one-way valves, in order to propel lymph forward against an unfavorable hydrostatic gradient and to prevent backflow (3-6). This coordination is achieved by intercellular transmission of contractile and dilatory signals throughout the lymphatic vessel wall, which is achieved by formation of connexin gap junctions between adjacent cells that mediate electrical coupling.

Connexins are a family of more than $\mathbf{2 0}$ proteins that form intercellular gap junctions between adjacent cells to allow and regulate ion and small molecule exchange. Connexins play an important role in the myelination of axons within the central nervous system (CNS) (7). The severe pediatric neurological disease, Pelizaeus-Merzbacher-like disease is the result of connexin 47 (CX47) deficiency (8). In addition to their role within the CNS, connexins are important for coordinating the smooth-muscle mediated contractility which propels lymphatic fluid centripetally (10-12) . In mice, four connexin proteins have been found to be expressed in lymphatic vessels: $\mathrm{Cx} 37, \mathrm{Cx} 43, \mathrm{Cx} 45$ and $\mathrm{Cx} 47(9,10,12)$. Interestingly, mouse $\mathrm{Cx} 47$ protein expression was found to be 5-times greater in lymphatic endothelial cells than in endothelial cells from blood vessels (13), and previous studies have reported missense $C X 47$ mutations cosegregating with primary lymphedema in humans $(14,15)$. In particular, two unique missense mutations located in the extracellular loop domains of CX47, R260C and S48L, were identified in two large families with dominantly inherited primary lymphedema, suggesting that specific mutations in CX47 may affect lymphatic function. Thus, to test the hypothesis that mutations in $C X 47$ lead to impaired lymphatic development and function, we used CRISPR-Cas9 technology to generate a mouse strain containing a mutation in the mouse $\mathrm{Cx} 47$ protein corresponding to the $C X 47 R 260 C$ mutation observed in humans with primary lymphedema.

MATERIALS AND METHODS

\section{Animals}

Mice were housed in clean cages and maintained on a 12-h light/dark schedule with free access to food (NIH-31 7913, Envigo Teklad, Indianapolis, IN) and distilled water. All animal procedures, including generation of transgenic strains, husbandry and experimental protocols, were approved by the University of Arizona's Institutional Animal Care and Use Committee and conformed with the Guide for the Care and Use of Laboratory Animals, $8^{\text {th }}$ edition, from the National Research Council.

\section{Generation of Cx47R259C Mutant Mice}

We used CRISPR technology in C57BL/ $6 \mathrm{~J}(\mathrm{~B} 6 / \mathrm{J})$ mice to alter the mouse $C_{x} 47$ gene sequence (also referred to as $\mathrm{Gjc} 2$ ) to produce a single amino acid substitution equivalent to the human $C X 47 R 260 C$ protein mutation previously reported (14). Due to sequence differences between species, the location of the equivalent arginine in the mouse protein is $\mathrm{R} 259$, thus the mouse mutation is designated $C x 47 R 259 C$. In addition to altering the mouse $C \times 47$ gene to produce the $\mathrm{Cx} 47 \mathrm{R} 259 \mathrm{C}$ mutant protein, a silent mutation introducing a BaeG1 restriction site was inserted in the mutant gene during the CRISPR process for purposes of genotyping the $C_{x} 47 R 259 C$ mice (Fig. 1A).

\section{Genotyping Protocol}

Genomic DNA was extracted and the region surrounding the $C x 47$ mutation and nearby BaeGI restriction site was amplified (KAPA HS Mouse Genotyping kit, Kappa Biosystems, Wilmington, MA) using the following primers: forward: 5'-GCAAGACGGTGGTCACTCC and reverse: 5'-GCCATCTCACAGAGGTTGAGC. PCR amplification of the wildtype gene generates a single $304 \mathrm{bp}$ product that is unaffected by $B a e G 1$ digestion (per manufacturer, NEB, Ipswich, MA), while amplification of the $C x 47 R 259 C$ mutant gene, , followed by $B a e G 1$ digestion, produces 2 bands (222 bp and $82 \mathrm{bp}$ ). Finally, amplification of DNA isolated from heterozygous mice, 
A. Cx47 Target Sequence

Cx47 wildtype sequence (bolded nucleotides were replaced by CRISPR)

TCGTGTCGCGgCCGACCGAGAAGACGGTCTTCTTGCT

CX47 mutant sequence (new nuceotides are bolded in red):

TCGTGTCGTGCCCTACCGAAAAGACAGTCTTCTTGCT

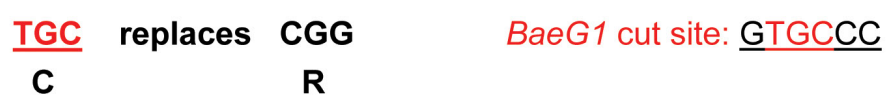

B. DNA Gel

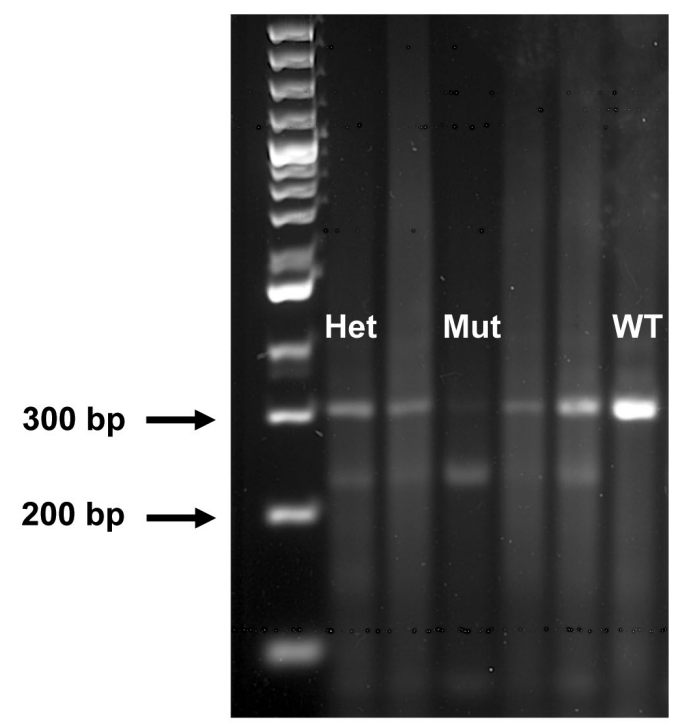

Fig. 1. Mouse wildtype Cx47 sequence and expected CRISPR mediated changes to the sequence to create the CX47R259C protein mutation. (A) Wildtype $C \times 47$ target sequence with planned changes needed to produce the CX47R259C protein, as well as the BaeG1 cut site. (B) DNA gel of BaeG1 digested PCR products from mouse tail DNA.

followed by $B a e G 1$ digestion, produces 3 bands (304 bp, 222 bp, and 82 bp). Following digestion, PCR products were run on a $1.5 \%$ agarose/TBE gel containing GelRed for detection ( $1 \mu \mathrm{l} \mathrm{GelRed} / 10 \mathrm{ml}$ agarose gel (VWR)). The 82 bp band was not routinely detected, thus genotypes were distinguished as follows: Wildtype (Wt): single 304 bp band, Heterozygous (Het): 304 bp and 222 bp bands, and Mutant (Mut): single 222 bp band (Fig. 1B).

\section{RNA Isolation and cDNA Preparation and Sequencing}

Total RNA extracted from mouse lymph nodes (Isolate II RNA Mini Kit, Meridian Life Science, Memphis, TN) was used to synthesize cDNA (Sensifast cDNA Synthesis kit, Meridian Bioscience). The heavily GC-rich coding region surrounding the $C X 47 R 259 C$ mutation was amplified (Kapa2G HS RM kit, Kapa 
Biosystems) and submitted for sequencing (Eton Bioscience, San Diego, CA).

\section{Lymphatic Phenotype}

Mice were weighed, anesthetized, placed on a warming pad, and examined grossly under a dissecting microscope (Weck, Evergreen, CO, USA) for external physical characteristics indicative of lymphatic abnormalities, i.e., distal edema, distichiasis, ocular abnormalities, and serous effusions, followed by Evans blue dye (EBD) injection. EBD ( $<50 \mu 1$ of 0.5 $\mathrm{g} / \mathrm{dl}$ ) was sequentially injected intradermally into the ear, hind paws, fore paws, and snout. Sequential dissection of the peritoneal, thoracic, axillary, jugular, popliteal, and sacral regions revealed EBD-stained lymphatic vessels, regional lymph nodes, cisterna chyli, and thoracic duct. Briefly, EBD was followed from the lumbar region proximally along the bifurcation of the aorta and inferior vena cava to the cisterna chyli. The mesentery was reflected and the usually milky, chyle-containing lymphatic trunks traced through the diaphragm into the thoracic cavity, where they formed the thoracic duct. The thoracic cavity was then opened and the presence of EBD in the TD was noted, if present, along with any abnormal dye reflux into intercostal lymphatic vessels. The duct was visualized traveling alongside the azygous vein until its final entry into the left subclavian vein. If grossly apparent, evidence of abnormal dye reflux into hindlimb skin or mesenteric lymphatic or lymph nodes was noted. EBD injected into the ear visualized the dermal lymph network.

The following were noted in each region: number of nodes, lobes per node, filled vs. unfilled nodes, presence of extra afferent and/or efferent lymphatic vessels and the presence of branched vessels.

In addition, the presence of chylous mesenteric reflux was determined in a subset of mice by dynamic evaluation under a dissecting microscope. In these mice, the peritoneum was opened and observed prior to injection of EBD. The chyle-containing lymphatic trunks were traced, and the cisterna chyli, renal artery, and mesenteric lymphatics and blood vasculature were identified and exposed. EBD was injected intradermally into both hind paws and its transport upwards through the lymphatics was monitored intra-abdominally, especially for mesenteric reflux. Mice were then sequentially injected with EBD in the ear, fore paws, and snout and regionally dissected as described above.

\section{Isolation and Cannulation of Superficial Cervical Lymphatic Vessels}

Mice were euthanized and placed in a dissection dish containing a sylgard pad and filled with calcium-free physiological salt solution (in $\mathrm{mM}: \mathrm{NaCl} 140, \mathrm{KCl} 5, \mathrm{MgCl}_{2} 2$, Dextrose 4, HEPES 10) supplemented with $0.1 \%$ bovine serum albumin (BSA, Fraction V heat-shock treated, Fisher Scientific, Fair Lawn, NJ). A midline incision was made in the skin from the top of the sternum to the lower mandible to expose the underlying tissue. Skin was retracted and afferent superficial lymphatic vessels were identified, running along the facial vein and terminating at the superficial lymph nodes located anterior to the thyroid gland. Afferent lymphatic vessels were carefully dissected to remove connective tissue and transferred to a second dissection dish containing Krebs buffer (in mM: $\mathrm{NaCl}$ 146.9, $\mathrm{KCl}$ 4.7, $\mathrm{CaCl}_{2} 2, \mathrm{MgSO}_{4} 1.2, \mathrm{NaH}_{2} \mathrm{PO}_{4}$ 1.2, $\mathrm{NaHCO}_{3} 3$, NaHEPES 1.5, Dextrose 5, pH 7.4) and were pinned down using minutien pins (Cat\# 26002-10, Fine Science Tools, Foster City, CA) for further removal of perivascular connective tissue. Vessels were transferred to a custom made $5 \mathrm{ml}$ tissue bath chamber filled with Krebs, both vessel ends were cannulated onto Krebs-filled small glass micropipettes $(50-70 \mu \mathrm{m}$ inner diameter) and then vessel plus micropipettes were moved to the stage of an inverted microscope (EXI-310PL, Accu-Scope, Commack, NY) equipped with a monochrome CCD camera (VesCam, IonOptix, Westwood, MA). Vessel diameter was continuously recorded at a frame rate of $15 \mathrm{~Hz}$ using the IonWizard software (IonOptix). Intraluminal pressure was set to $3 \mathrm{~cm}$ $\mathrm{H}_{2} \mathrm{O}$ using a custom-made standing water reservoir. Vessels were equilibrated with Krebs 
solution $\left(37^{\circ} \mathrm{C}\right)$ at a constant exchange rate of $\sim 1 \mathrm{ml} / \mathrm{min}$ until a stable rhythmic contraction frequency was generated (30-45 minutes total equilibration time). This is the baseline contractile frequency.

\section{Pressure Curve}

Following equilibration, lymphatic vessel intraluminal pressure was decreased to $1 \mathrm{~cm}$ $\mathrm{H}_{2} \mathrm{O}$ for 3 minutes and then increased to $2 \mathrm{~cm}$ $\mathrm{H}_{2} \mathrm{O}$ for 3 minutes. This stepwise increase was repeated for 3, 5, 7 and $10 \mathrm{~cm} \mathrm{H}_{2} \mathrm{O}$, with 3-minute recordings at each pressure. This protocol promotes a graded increase in contraction frequency, together with a decrease in contractile amplitude (16).

Following the 3-minute recording at $\mathbf{1 0}$ $\mathrm{cm} \mathrm{H}_{2} \mathrm{O}$, intraluminal pressure was returned to $3 \mathrm{~cm} \mathrm{H}_{2} \mathrm{O}$ and the contractile frequency was compared to that of the baseline contractile frequency. Lymphatic vessels showing more than $20 \%$ reduction in post-pressure curve contractile frequency were discarded. The following parameters were analyzed: 1) Contractile frequency (number of contractions per minute); 2) End-diastolic diameter (EDD, maximum lumen diameter immediately after the start of contraction); 3) End-systolic diameter (ESD, diameter at peak contraction); and 4) Ejection fraction, defined as a fractional reduction in luminal area at each contraction and calculated as EF $=\left(\mathrm{EDD}^{2}-\mathrm{ESD}^{2}\right) /$ $\operatorname{EDD}^{2}(4)$.

\section{STATISTICAL ANALYSES}

All statistical analyses were performed using GraphPad Prism v.7 or v.8 (GraphPad Software, San Diego, CA).

Lymphatic phenotype data were analyzed by two-way ANOVA (GraphPad Prism v.7), with a Dunett's post-hoc correction for multiple comparisons when overall group effects were found to be significant. Differences between group means with a value of $p<0.05$ were considered statistically significant. Pressure myography data were analyzed by two-way ANOVA for all parameters assessed during the pressure curve (contractile frequency and EF), with a Sidak post-hoc correction for multiple comparisons. All data are expressed as means \pm S.E.M.

\section{RESULTS}

\section{Confirmation of Cx47R259C and Wildtype mRNA in Lymph Nodes}

To confirm transcription of the mutant $C_{x} 47 R 259 C$ and wildtype $C_{x} 47$ genes within lymphatic tissues, total RNA was isolated from regional lymph nodes (iliac, jugular, and popliteal) and used to generate cDNA for Sanger sequencing (Eton Biosciences, San Diego, CA). cDNA sequencing confirmed that the mutant $C x 47 R 259 C \mathrm{mRNA}$ was expressed in the iliac, jugular and popliteal lymph nodes of homozygous $C x 47 R 259 C$ mice. Heterozygous mice expressed both mutant ( $C x 47 R 259 C)$ and wildtype $C x 47 \mathrm{mRNA}$, while wildtype littermates expressed only the wildtype $C_{x} 47$ mRNA (Fig. 1 and $2 A-C$ ).

\section{Phenotypes}

External gross examination under a dissecting microscope was carried out on all mice prior to EBD injection. All mice were phenotypically normal by gross examination, irrespective of genotype.

Intradermal EBD injection showed plexiform branching of the ear lymphatics in homozygous $C x 47 R 259 C$ mice but not in the ears of wildtype mice (Fig. 3A-B). Intradermal EBD injection, followed by sequential dissection (described in Methods), was used to visualize lymph nodes and lymphatic vessels. The lymphatic phenotypic features determined in this study, i.e., number of nodes, lobes per node, number of vessels, and presence of branched vessels, did not differ between heterozygous and wildtype mice (Fig. 4). All homozygous $C X 47 R 259 C$ mice exhibited increased numbers of total lymph nodes in one or more of the following regions when compared to wildtype: jugular $(4.8 \pm 0.4$ vs $2.0 \pm 0, \mathrm{p}<0.05$, Fig. $4 A, D, G)$, cisterna chyli $(1.8 \pm 0.2$ vs 1.0 


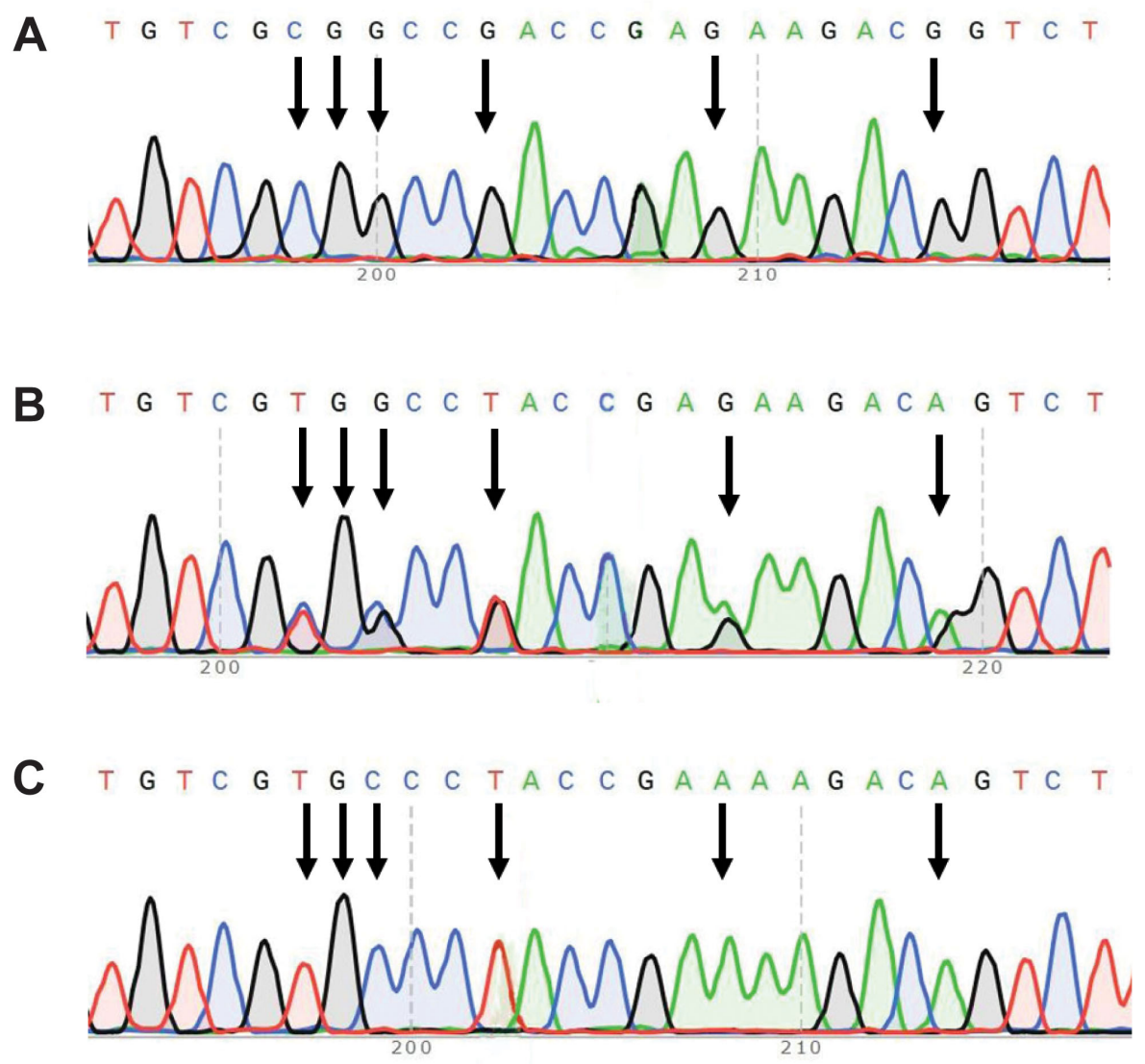

Fig. 2. Sanger sequencing of lymph mode cDNA collected from wildtype, heterozygous CX47R259C, and homozygous CX47R259C mice. A-C) Representative sequences of CX47 gene (Gcj2) cDNA of a lymph node isolated from wildtype (A), heterozygous (B) and homozygous CX 47R259C mutant (C). The arrows point to nucleotides altered in one of the alleles of heterozygous mice and altered in both alleles of Cx47R259C mice.

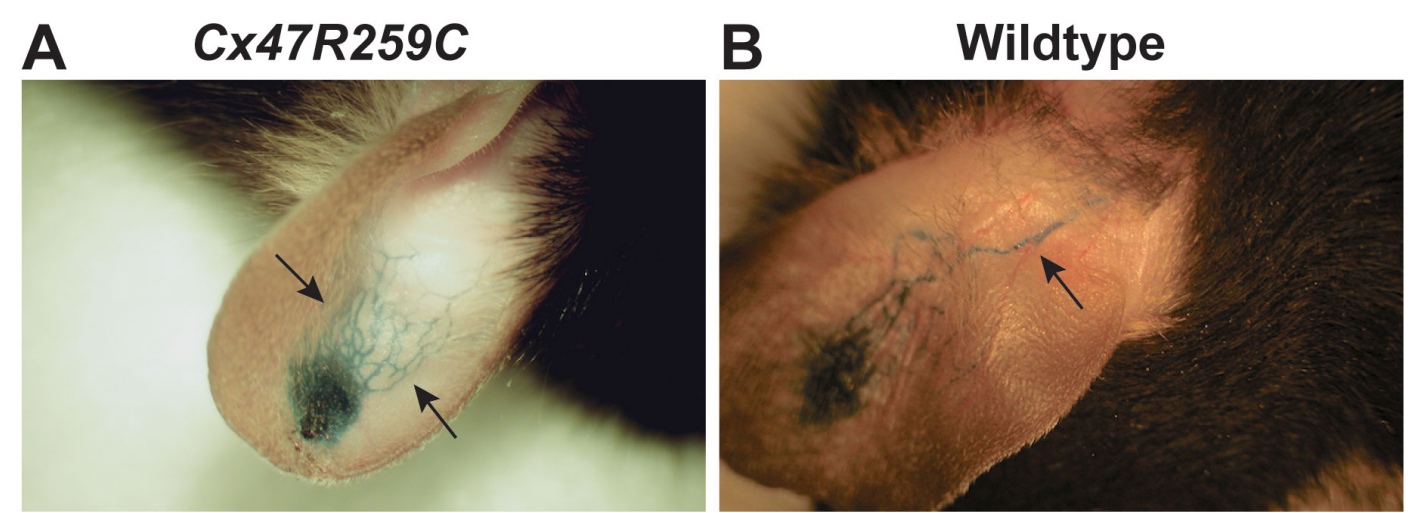

Fig. 3. Dermal lymphatic vessels following intradermal injection of Evans blue dye (EBD). (A) Presence of plexiform branching (black arrows) in a homozygous CX47R259C mouse ear and (B) absence of plexiform branching and presence of a central draining lymphatic (black arrow) in the ear of a wildtype mouse. 

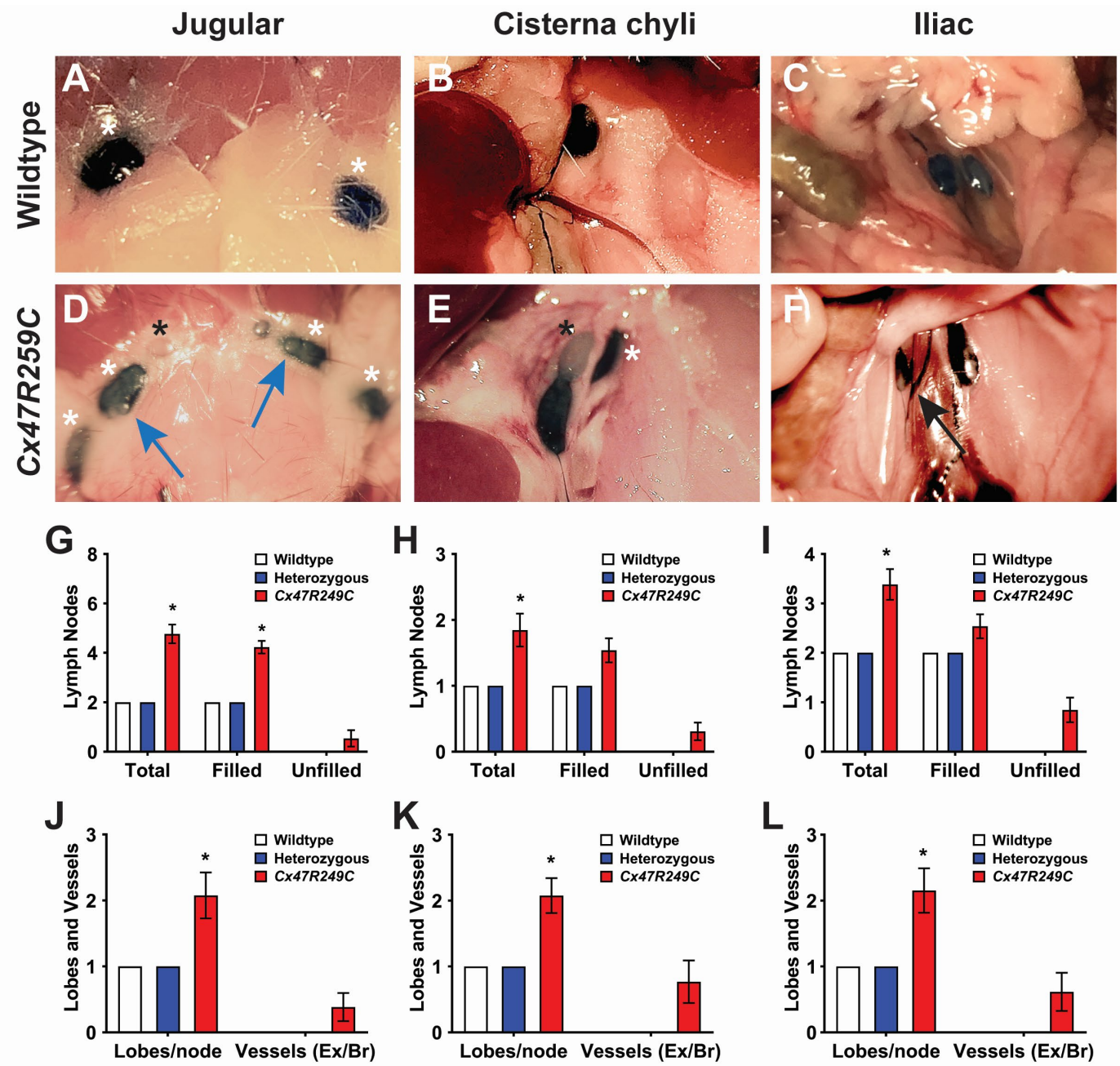

Fig. 4. Lymph nodes and lymphatic vessels in wildtype and homozygous CX $47 R 259 C$ mice following intradermal injection of EBD. Homozygous CX47R259C mice have increased EBD-filled (white *) and EBD-unfilled (black*) lymph nodes, extra lobes per lymph node (blue arrows), and increased and/or branched lymphatic vessels (black arrows) compared to wild-type mice. Wildtype: $(A)$ jugular, $(B)$ cisterna chyli, and $(C)$ iliac regions compared to homozygous CX47R259C mice (D-F). EBD was injected intradermally into hind paws, fore paws, and snout followed by sequential dissection. $G$-L) Summary data showing increased number of lymph nodes $(G-I)$ in CX 47R259C mice, as well as number of lobes per node and number of lymphatic vessels (J-L). Ex/Br: extra I branched lymphatic vessels. Data are means \pm S.E.M, $*=p<0.05$, compared to wildtype, two-way ANOVA. $n=5$ for both wildtype and heterozygous, and $n=13$ for Cx47R259C homozygous mutants.

\pm 0 . p $<0.05$, Fig. $4 B, E, H)$ and iliac $(3.4 \pm 0.3$ vs $2.0 \pm 0$, p $<0.05$, Fig. $4 C, F, I)$. In addition, the average number of lobes per node in each of these three regions was 2.1-fold greater in homozygous $C_{x} 47 R 259 C$ than in wildtype mice (p<0.05, Fig. 4J-L). Furthermore, increased vessels and/or branched vessels were present in the iliac, jugular, and cisterna chyli regions of 8/13 homozygous $C x 47 R 259 C$ mice but were not present in wildtype mice (Fig. 4J-L). The presence of extra and/or branched vessels was particularly pronounced in the iliac region of homozygous $C x 47 R 259 C$ mice as compared to wildtype mice (Fig. 4J-L). Although the 


\section{Pre-injection}

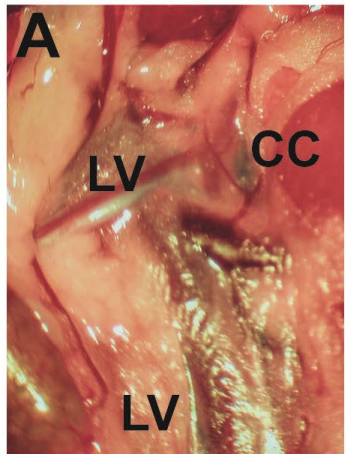

$3 \min 15 \mathrm{~s}$

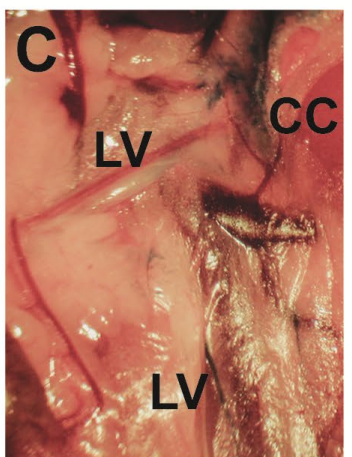

\section{$3 \mathrm{~min}$}

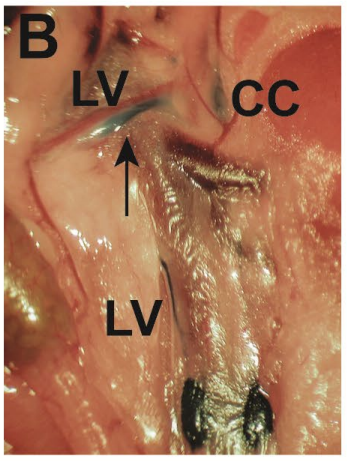

$3 \mathrm{~min} 30 \mathrm{sec}$

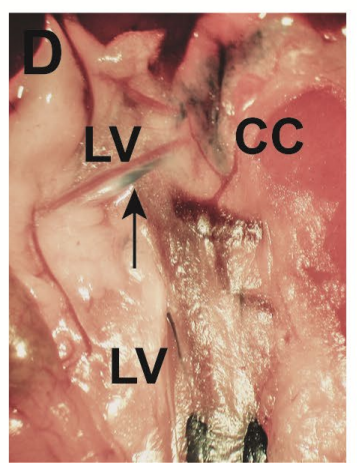

E

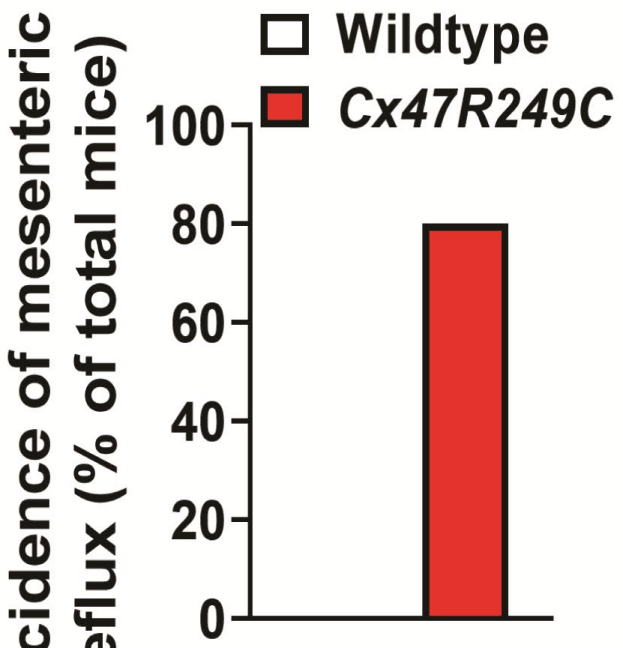

Fig. 5. Dynamic imaging of chylous mesenteric reflux in a homozygous Cx47R259C mouse following intradermal injection of Evans blue dye (EBD) into the hind paws. The peritoneal cavity was opened prior to intradermal injection of EBD to allow observation of reflux from the cisterna chyli (CC) to the lymphatic vessel $(L V)$. The site of reflux is evidenced by the black arrow. (A) Pre-injection, (B) 3 min post injection, (C) 3 min 15 $\mathrm{sec}$, (D) 3 min $30 \mathrm{sec}$. (E) Summary data showing incidence of chylous mesenteric reflux in Cx47R259C mice.

presence of $C_{x} 47 R 259 C \mathrm{mRNA}$ was confirmed in the popliteal and sacral lymphatic nodes of homozygous $C x 47 R 259 C$ mice, no phenotypic alterations were observed in these areas. Similarly, no anatomical differences were observed in thoracic ducts of homozygous $C x 47 R 259 C$ mice when compared to wildtype mice.

Dynamic EBD evaluation of lymphatics demonstrated chylous mesenteric reflux in 4/5 homozygous $C x 47 R 259 C$ mice tested for reflux (Fig. $5 A-D$ ) but was not seen in wildtype mice (summary data in Fig. 5E).

\section{Lymphatic Vessel Contractility}

To test the hypothesis that a missense Cx47R259C mutation leads to impaired contractile function in lymphangions, superficial cervical lymphatic vessels were isolated and cannulated on a pressure myograph system and subjected to increasing intraluminal pressures. We observed a pressure-dependent increase in contractile frequency in lymphatic vessels with the increase in intraluminal pressures for both wildtype and homozygous Cx47R259C mice (Fig. 6A). However, the increase in frequency was significantly attenuated in homozygous $C X 47 R 259 C$ mice when compared to wildtype mice (Fig. $6 B, C$ ), to the point where frequency was lower in mutants 
A
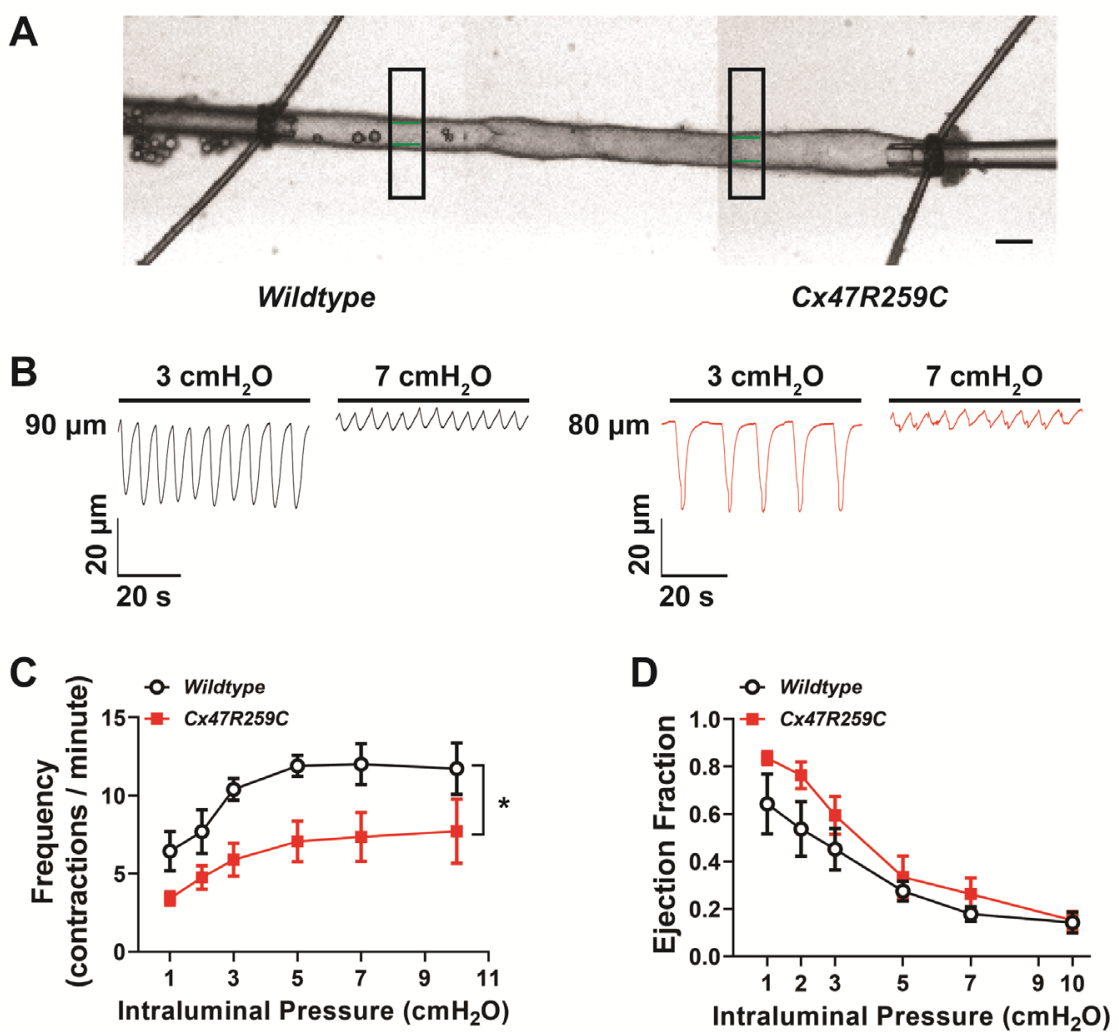

Fig. 6. Reduced contractility in superficial cervical lymphatic vessels of homozygous Cx47R259C mice compared to wildtype mice. (A) Representative image of an isolated superficial cervical lymphatic vessel showing 1 valve and 2 inter-valve segments. The black boxes represent areas of real-time lumen diameter measurements. Bar $=50 \mu \mathrm{m}$. (B) Representative traces of the lumen diameter of lymphatic vessels isolated from wildtype (black traces, left) and homozygous Cx47R259C mice (red traces, right) at 3 and $7 \mathrm{~cm} \mathrm{H2O.} \mathrm{(C)} \mathrm{Summary} \mathrm{data} \mathrm{showing} \mathrm{a}$ significant decrease in contractile frequency in lymphatic vessels from homozygous Cx47R259C mice (red) facing step-wise increases in intraluminal pressure when compared to vessels from wildtype mice. * $p<0.05$, two-way ANOVA with a Sidak correction for multiple comparisons, $n=5$ vessels from 5 different mice for each genotype. (D) Summary data showing that ejection fraction was not significantly altered by the CX47R259C missense mutation.

throughout the entire pressure curve. Ejection fraction, or the fractional reduction in diameter observed at each contraction, was slightly higher in homozygous $C x 47 R 259 C$ mice than in wildtype at the pressures of 1 and $2 \mathrm{~cm}$ $\mathrm{H}_{2} \mathrm{O}$, although no differences were statistically significant (Fig. 6D).

\section{DISCUSSION}

Connexins, the proteins that form gap junctions between adjacent cells, have a clear role in lymphatic physiology and development. Connexins $\mathrm{Cx} 37, \mathrm{Cx} 43$, and $\mathrm{Cx} 47$ are expressed in the lymphatic endothelial cells and are enriched at lymphatic valves (10). $\mathrm{Cx} 47$, in particular, is also found in endothelial cells of veins, with wide expression early in development, then becoming restricted to venous valves (17). Genetic ablation of $\mathrm{Cx} 37$ and $\mathrm{Cx} 43$ leads to lymphatic valve malformations, resulting in embryonic lymphedema and chylothorax with markedly reduced post-natal survival (10). Similarly, deletion of Cx37 
prevents valve formation in murine veins (18). However, genetic deletion of $\mathrm{Cx} 47$ in lymphatic endothelial cells did not induce developmental lymphatic malformations (except when combined with $\mathrm{Cx} 43$ deficiency (17), or contractility deficits in mice $(9,19)$.

Mutations in the human $C X 47$ gene (also called $G J C 2$ ) are associated with recessive disorders, such as Pelizaeus-Merzbacher-like disease, a type of recessive hypomyelination leukodystrophy that causes severe lack of myelination in the white matter, accompanied by motor and cognitive deficits (20). To date more than $\mathbf{5 0}$ mutations have been found in the $C X 47$ gene, many of them leading to Pelizaeus-Merzbacher-like disease of different severities $(21,22)$. Interestingly, the severity of the disorder seems to be linked to the location of the mutation and/or the resulting type of structural alteration caused by the mutation, i.e., missense mutations resulting in truncated or non-functional CX47 proteins, due to the generation of an early stop codon, are associated with more severe disease (20). In addition to neurological disorders, mutations in the CX47 gene have been linked to primary lymphedema $(14,15)$ and increased risk of developing secondary lymphedema after treatment for breast cancer (23).

Missense mutations leading to a single nucleotide change located in the extracellular domains of the CX47 protein are associated with dominantly inherited primary lymphedema in humans. Two such missense mutations (R260C and S48L) were described in two non-related families (14). Each mutation cosegregated with primary lymphedema in the family carrying that particular mutation, with affected individuals from both families reporting "uncomplicated lymphedema of the leg or hand" as a consistent feature. Only one family member with the R260C mutation was non-penetrant for lymphedema. These findings were followed by the discovery of another novel lymphedema-inducing CX47 missense mutation (M210R) in a family with primary lymphedema, as well as four additional families with primary lymphedema cosegregating with the S48L missense mutation (15). Importantly, lymphoscintigraphy in members of these families suggested a functional deficit rather than anatomical abnormalities (15).

In our study using a CRISPR-generated mouse with a single missense mutation $(C x 47 R 259 C)$ that is equivalent to the human $C X 47 R 260 C$ mutation associated with primary lymphedema, we observed anatomical alterations of lymphatic vessels and nodes, impaired contractile function of collecting lymphatic vessels, and mesenteric reflux in mice homozygous for the $C x 47 R 259 C$ mutation. This suggests that, both in humans and mice, the $C \times 47 R 259 C / C X 47 R 260 C$ is an altered function mutant. In support of a functional deficit, we observed that superficial lymphatic vessels isolated from homozygous $C_{x} 47 R 259 C$ mice showed reduced contractile frequency when compared to vessels isolated from wildtype littermates, as well as chylous mesenteric reflux after hindpaw EBD injection. This finding is in contrast to data from previous reports using $\mathrm{Cx} 47$ knock-out mice indicating that $\mathrm{Cx} 47$ deletion has little or no effect on the contractility of popliteal afferent (9) or inguinal (19) collecting lymphatic vessels. The underlying reason for the differences observed requires further study but may be a consequence of: 1) the different genetic manipulations applied in the studies, i.e., deletion of the protein vs. expression of a mutant protein with a single amino acid substitution and/or 2) location-specific expression of different connexin isoforms. The constitutive deletion of $C \times 47$ in earlier studies may have induced overexpression of other isoforms present in lymphatic endothelial cells, such as $\mathrm{Cx} 37$ and $\mathrm{Cx} 43$, to maintain normal lymphatic function. It is possible that a missense mutation in $\mathrm{Cx} 47$, such as the one generated in the present study and expected to result in expression of mutant protein, does not alter expression levels of Cx37 and/or Cx43 and thus no compensation occurs in our mice or in humans with the $C X 47 R 260 C$ mutation. Rather, expression of a $\mathrm{Cx} 47$ protein with a mutation replacing the positively charged arginine at position 260 with a non-charged polar cysteine is expected to prevent formation of an essential "salt-bridge" between Cx47 extra- 
cellular loops E1 and E2. This salt bridge is crucial to maintaining loop 2 in the correct position for the connexin-connexin interaction needed to generate functional connexons (21). Thus, it will likely affect formation of functional gap junction channels as it is located in the highly conserved SRPTEK motif of extracellular loop 2 which is crucial for docking of adjacent connexin hemichannels and formation of functional pores $(24,25)$. More specifically, based on molecular modeling studies, it is possible to speculate that the substitution of a cysteine (C) for an arginine (R) may create an imbalance in the charges between the two Cx47 extracellular loops, consequently altering the position of loop 2 and preventing the docking of adjacent connexin hemichannels and formation of a fully functional gap junction between lymphatic endothelial cells. Consequently, the lack of functional $\mathrm{Cx} 47$ connexons may lead to contractile deficits and the lymphatic phenotypes observed in $C x 47 R$ $259 C$ mice. Lastly, Cx47 immunoreactivity was not detected in the popliteal afferent vessels of either knockout or wildtype mice, thus a lack of regional protein expression may explain the lack of functional effects caused by $\mathrm{Cx} 47$ deletion (9). In agreement with this possible location-specific expression, we did not observe any anatomical abnormalities in popliteal vessels or nodes in the $C x 47 R 259 C$ mice.

In general, mutations in genes related to defective valve formation tend to produce lymphatic hyperplasia/lymphangiectasia and lymph reflux. In contrast, the genes involved in initiation or maintenance/proliferation produce primary lymphedema with lymphatic hypoplasia/aplasia in the periphery and even centrally. In general, genetic mouse models of human mutations causing lymphedema do not show peripheral lymphedema. This may reflect the shorter distance for ISF to travel through tissues or the fact that most human genetic lymphedema is not present at birth and appears later, especially at puberty in females. However, the hyperplastic lymphatic phenotype we report was found with other mouse models with defective lymphatic valve formation, especially the Foxc2 knockout (26) and Foxc 2 over-expressing mice (27). This is a hyperplastic, refluxing, valve-incompetent lymphatic phenotype with lymphatic collector vessels and lymph nodes increased in number in the inguinal, retroperitoneal, axillary and cervical regions. This hyperplastic lymphatic phenotype is less common than the hypoplastic form seen in typical Milroy disease and other primary lymphedemas, e.g., associated with VEGFR3 mutations, and has also been described in patients with some rarer forms of primary lymphedema, notably lymphedemadistichiasis, FOXC2 deficiency (28). This hyperplasia can involve both increased numbers of lymphatic vessels as well as increased number of regional lymph nodes demonstrable in the inguinal and retroperitoneal area of primary lymphedema patients on conventional oil contrast lymphography (28). This seems also to be true in humans as exemplified by a patient with full-blown lymphedema-distichiasis with two copies (i.e., an extra duplicated copy) of the mutant FOXC2 gene and striking lymphatic hyperplasia associated with extensive lymph refluxing and lymphatic valve incompetence (29). Mesenteric lymph node architecture in the Foxc2 deficient mouse shows a characteristic dysmorphology (30) as did lymph node examination in the previously described patient with overexpression of FOXC2(29).

The similarity in phenotype probably reflects the involvement of Foxc2 upstream of connexins, there are connections between FOXC2 control and the lymphatic endothelial expression of connexins in a common signaling pathway - where $F O X C 2$ controls the expression of lymphedema-causing Connexin 37, 43, and 47 (as well as CELSR1) all related to initial lymphatic valve development and/or secondary disrupted/maldeveloped/incompetent valves caused by more proximal lymphatic obstruction/maldevelopment/lymphangiodysplasia (12).

The reduced contractile activity of collecting lymphatic vessels may underlie the anatomical lymphatic abnormalities observed in $C x 47 R 259 C$ mice. We observed excessive branching and augmented number of lymphatic vessels and nodes in the iliac, jugular and axillary regions as well as in the cisterna 
chyli. It is possible that formation of new lymphatic vessels is a consequence of impaired tissue lymphatic drainage, with the organism overcompensating by generating collateral lymphatic vessels. It is also possible that these anatomical differences caused by the $C \times 47 R$ $259 \mathrm{C}$ missense mutation are a consequence of other developmental issues. This is a possibility that requires further future study. The $C x 47 R 259 C$ mouse is expected to provide a much needed model to further interrogate the role of $\mathrm{Cx} 47$ in the normal lymphatic system as well as in primary lymphedema.

In summary, we observed that a $\mathrm{Cx} 47$ missense mutation $(C x 47 R 259 C)$, an amino acid exchange similar to that observed in humans with primary lymphedema, induces anatomical abnormalities in lymphatic vessels and nodes of select regions as well as functional deficits observed as impaired contractility of collecting lymphatics and chylous mesenteric reflux. Further studies are needed to identify the molecular and cellular mechanisms underlying the functional alterations as well as to determine if anatomical changes are caused by developmental malformations or occur postbirth to overcome impaired lymphatic drainage of tissues. The use of more relevant preclinical models that replicate human genetics are imperative to determining the functional role(s) for the various protein mutations found to be associated with primary lymphedema, including CX47 and its associated mutations and are fundamental to the development of future therapeutic regimens for this debilitating disease.

\section{ACKNOWLEDGMENTS}

The present study was supported by funds from the National Heart, Lung and Blood Institute (R00HL140106 to PWP, R25HL108837 and T35HL007479 to MHW and RJM; R21HL122443 to AMS, MHW, and JDK), National Institute of Neurologic Disorders and Stroke (R25NS076437 to MHW, JAJ and RJM), National Institute of Childhood Health and Human Development (R25HD080811 to MHW and RJM), the Western Alliance to Expand Student Opportunities
(WAESO) Louis Stokes Alliance for Minority Participation (LSAMP) from the National Science Foundation (HRD-1619524 to RJM and JAJ), and intramural Translational Imaging Program Projects Stimulus Funds to MHW. Production of the CRISPR mouse by the University of Arizona Genetically Engineered Mouse Models Core was supported by the National Cancer Institute of the National Institutes of Health under award number P30 CA023074. We thank the University of Arizona Cancer Center's EMSR Laboratory (supported by National Cancer Institute of the National Institutes of Health award number P30 CA023074) and Jalicia Washington (supported by National Heart, Lung and Blood Institute R25 HL108837) for colony management. The funding agencies had no responsibilities on the conception, design and analysis of experimental data in this manuscript.

\section{CONFLICT OF INTEREST AND DISCLOSURE}

The authors declare no competing financial interests exist.

\section{REFERENCES}

1. Executive Committee of the International Society of Lymphology: The diagnosis and treatment of peripheral lymphedema: 2020 Consensus Document of the International Society of Lymphology. Lymphology 53 (2020), 3-19.

2. Grada, AA, TJ Phillips: Lymphedema: Pathophysiology and clinical manifestations. J. Am. Acad. Dermatol. 77 (2017), 1009-1020.

3. Rockson, SG, KK Rivera: Estimating the population burden of lymphedema. Ann. NY Acad. Sci. 1131 (2008), 147-154.

4. Scallan, JP, SD Zawieja, JA Castorena-Gonzalez, et al: Lymphatic pumping: Mechanics, mechanisms and malfunction. J. Physiol. 594 (2016), 5749-5768.

5. Eisenhoffer, J, A Kagal, T Klein, et al: Importance of valves and lymphangion contractions in determining pressure gradients in isolated lymphatics exposed to elevations in outflow pressure. Microvasc. Res. 49 (1995), 97-110. 
6. Schmid-Schonbein, GW: Microlymphatics and lymph flow. Physiol. Rev. 70 (1990), 987-1028.

7. Menichella, DM, DA Goodenough, E Sirkowski, et al: Connexins are critical for normal myelination in the CNS. J. Neurosci. 23 (2003), 5963-5973.

8. Uhlenberg, B, M Schuelke, F Ruschendorf, et al: Mutations in the gene encoding gap junction protein alpha 12 (connexin 46.6) cause Pelizaeus-Merzbacher-like disease. Am. J. Hum. Genet. 75 (2004), 251-260.

9. Castorena-Gonzalez, JA, SD Zawieja, M Li, et al: Mechanisms of connexin-related lymphedema. Circ. Res. 123 (2018), 964-985.

10. Kanady, JD, MT Dellinger, S Munger, et al: Connexin 37 and Connexin 43 deficiencies in mice disrupt lymphatic valve development and result in lymphatic disorders including lymphedema and chylothorax. Dev. Biol. 354 (2011), 253-266.

11. Kanady, JD, AM Simon: Lymphatic communication: Connexin junction, what's your function? Lymphology 44 (2011), 95-102.

12. Sabine, A, Y Agalarov, H Maby-El Hajjami, et al: Mechanotransduction, PROX1, and FOXC2 cooperate to control connexin 37 and calcineurin during lymphatic-valve formation. Dev. Cell 22 (2012), 430-445.

13. Wick, N, P Saharinen, J Saharinen, et al: Transcriptomal comparison of human dermal lymphatic endothelial cells ex vivo and in vitro. Physiol. Genomics 28 (2007), 179-192.

14. Ferrell, RE, CJ Baty, MA Kimak, et al: GJC2 missense mutations cause human lymphedema. Am. J. Hum. Genet. 86 (2010), 943-948.

15. Ostergaard, P, MA Simpson, G Brice, et al: Rapid identification of mutations in GJC2 in primary lymphoedema using whole exome sequencing combined with linkage analysis with delineation of the phenotype. J. Med. Genet. 48 (2011), 251-255.

16. Zawieja, SD, JA Castorena-Gonzalez, JP Scallan, et al: Differences in L-type $\mathrm{Ca}(2+)$ channel activity partially underlie the regional dichotomy in pumping behavior by murine peripheral and visceral lymphatic vessels. Am. J. Physiol. Heart Circ. Physiol. 314 (2018), H991-H1010.

17. Munger, SJ, X Geng, RS Srinivasan, et al: Segregated Foxc2, NFATc1 and Connexin expression at normal developing venous valves, and Connexin-specific differences in the valve phenotypes of $\mathrm{Cx} 37, \mathrm{Cx} 43$, and $\mathrm{Cx} 47$ knockout mice. Dev. Biol. 412 (2016), 173-190.
18. Munger, SJ, JD Kanady, AM Simon: Absence of venous valves in mice lacking Connexin37. Dev. Biol. 373 (2013), 338-348.

19. Meens, MJ, I Kutkut, V Rochemont, et al: Cx47 fine-tunes the handling of serum lipids but is dispensable for lymphatic vascular function. PLoS ONE 12 (2017), e0181476.

20. Owczarek-Lipska, M, L Mulahasanovic, CD Obermaier, et al: Novel mutations in the GJC2 gene associated with Pelizaeus-Merzbacher-like disease. Mol. Biol. Rep. 46 (2019), 4507-4516.

21. Biancheri, R, C Rosano, L Denegri, et al: Expanded spectrum of Pelizaeus-Merzbacher-like disease: Literature revision and description of a novel GJC2 mutation in an unusually severe form. Eur. $\mathbf{J}$. Hum. Genet. 21 (2013), 34-39.

22. Orthmann-Murphy, JL, AD Enriquez, CK Abrams, et al: Loss-of-function GJA12/Connexin47 mutations cause Pelizaeus-Merzbacher-like disease. Mol. Cell Neurosci. 34 (2007), 629-641.

23. Finegold, DN, CJ Baty, KZ Knickelbein, et al: Connexin 47 mutations increase risk for secondary lymphedema following breast cancer treatment. Clin. Cancer Res. 18 (2012), 2382-2390.

24. Dahl, G, W Nonner, R Werner: Attempts to define functional domains of gap junction proteins with synthetic peptides. Biophys. J. 67 (1994), 1816-1822.

25. Warner, A, DK Clements, S Parikh, et al: Specific motifs in the external loops of connexin proteins can determine gap junction formation between chick heart myocytes. J. Physiol. 488 (Pt 3) (1995), 721-728.

26. Kriederman, BM, TL Myloyde, MH Witte, et al: FOXC2 haploinsufficient mice are a model for human autosomal dominant lymphedema-distichiasis syndrome. Hum. Mol. Genet. 12 (2003), 1179-1185.

27. Noon, A, RJ Hunter, MH Witte, et al: Comparative lymphatic, ocular, and metabolic phenotypes of Foxc2 haploinsufficient and aP2-FOXC2 transgenic mice. Lymphology 39 (2006), 84-94.

28. Kinmonth, JB: The Lymphatics: Diseases, Lymphography and Surgery. Edward Arnold (1972).

29. Witte, MH, RP Erickson, M Khalil, et al: Lymphedema-distichiasis syndrome without FOXC2 mutation: Evidence for chromosome 16 duplication upstream of FOXC2. Lymphology 42 (2009), 152-160. 
30. Shimoda, H, MJ Bernas, MH Witte:

Dysmorphogenesis of lymph nodes in Foxc2

haploinsufficient mice. Histochem. Cell Biol.

135 (2011), 603-613.

Paulo W. Pires, PhD

Assistant Professor of Physiology, Neurosurgery, and Surgery

University of Arizona College of Medicine

1501 N. Campbell Avenue

Tucson, AZ 85724-5051

Telephone: (520) 626-8632

E-mail: ppires@email.arizona.edu 